\title{
On The Use of Machine Learning Algorithms to Classify Focal Cortical Dysplasia on MRI
}

\author{
João Guilherme Pereira, Matheus de Freitas Oliveira Baffa, Fabrício Henrique Simozo, \\ Luiz Otavio Murta Junior, Joaquim Cezar Felipe
}

\author{
Departamento de Computação e Matemática \\ Faculdade de Filosofia, Ciências e Letras de Ribeirão Preto \\ Universidade de São Paulo (USP) \\ Ribeirão Preto, SP - Brasil \\ \{joao.guilherme, mbaffa, fsimozo\}@usp.br, \{murta, jfelipe\}@ffclrp.usp.br
}

\begin{abstract}
Refractory epilepsy is a condition characterized by epileptic seizure occurrence which cannot be controlled with antiepileptic drugs. This condition is associated with an excessive neuronal discharge produced by a group of neurons in a certain epileptogenic zone. Focal Cortical Dysplasia (FCD), usually found in these zones, was detected as one of the main causes of refractory epilepsy. In these cases, surgical intervention is necessary to minimize or eliminate the seizure occurrences. However, surgical treatment is only indicated in cases where there is complete certainty of the FCD. In order to assist neurosurgeons to detect precisely these regions, this paper aims to develop a classification method to detect FCD on MRI based on morphological and textural features from a voxel-level perspective. Multiple classifiers were tested throughout the extracted features, the best results achieved an accuracy of $91.76 \%$ using a Deep Neural Network classifier and $96.15 \%$ with $J 48$ Decision Tree. The set of evaluating metrics showed that the results are promising.
\end{abstract}

Resumo. A epilepsia refratária é uma condição caracterizada pela ocorrência de crise epiléptica que não pode ser controlada com medicamentos antiepilépticos. Essa condição está associada a uma descarga neuronal excessiva produzida por um grupo de neurônios em uma determinada zona epileptogênica. A Displasia Cortical Focal (DCF), comumente encontrada nessas zonas, foi detectada como uma das principais causas de epilepsia refratária. Nestes casos, a intervenção cirúrgica é necessária para minimizar ou eliminar as ocorrências de convulsões. Porém, o tratamento cirúrgico só é indicado nos casos em que haja certeza absoluta da DCF. A fim de auxiliar os neurocirurgiões a detectar precisamente essas regiões, este artigo tem como objetivo desenvolver um método de classificação para detectar DCF em imagens de Ressonância Magnética com base em características morfológicas e texturais extraídas a partir da perspectiva de cada voxel. Para realizar tal tarefa, diversos métodos de aprendizado supervisionado foram testados utilizando a base de características extraídas. Os melhores resultados alcançaram uma acurácia de 91,76 \% usando um classificador baseado em Rede Neural Profunda e 96,15\% utilizando uma árvore de decisão J48. O conjunto de métricas de avaliação mostra que os resultados são promissores na solução do problema levantado. 


\section{Introduction}

Epilepsy is a neuronal disorder that causes epileptic seizures. Excessive discharges produced by a group of neurons causes this condition on patients with a wide range of ages [Fisher et al. 2014]. Epilepsy can be a transient disorder in which $70 \%$ to $80 \%$ of patients can control seizures with antiepileptic drugs (AEDs) [Kwan and Brodie 2000].

Epilepsy is a disease that affects people of all races, genders, socioeconomic conditions, and regions. It is estimated that 50 million people have epilepsy worldwide. Of these, 40 million live in developing countries [Gallucci Neto and Marchetti 2005]. According to the World Health Organization (WHO), two-thirds of patients affected by this disease do not receive the treatments indicated for disease control [WHO 2019].

Refractory Epilepsy, also known as pharmacoresistant epilepsy, is the occurrence of epileptic seizures that do not have AED-type drugs to control seizures. Patients with this type of disease suffer from frequent seizures which threaten their quality of life and can cause irreversible damage [Sheppard and Lippé 2012, Roy et al. 2011].

Recent studies show that the incidence of Refractory Epilepsy is high. One in every three patients diagnosed with epilepsy will develop pharmacoresistant epilepsy [Li and Hurford 2020, French 2007]. Also, considering the lack of continuous release of new antiepileptic drugs, approximately 20 to $40 \%$ of new patients diagnosed with epilepsy will become refractory [French 2007].

In this specific type of epilepsy, surgical intervention is necessary to minimize or eliminate its occurrence. However, surgical treatment is only indicated in cases where there is a complete certainty of the epileptogenic zone, that is, the region that causes excessive neuronal discharges. The complete resection or disconnection of this area leads to the interruption of the crises [Rosenow and Lüders 2001].

Within the scope of epileptogenic lesions, a group of lesions named Focal Cortical Dysplasia (FCD) was detected as one of the main causes of Refractory Epilepsy. This type of lesion has heterogeneous histopathological characteristics, with clinical presentation and distinct images [Taylor et al. 1971, Colombo et al. 2003].

FCD are difficult to diagnose lesions and, even when identified, determining their limits is usually a hard task. Currently, several works in the literature detect these lesions on Magnetic Resonance Images (MRI) [Antel et al. 2003, Hong et al. 2014, Wang et al. 2020] however, they are not easily available for use. Thus, we emphasize the importance of developing computer-aided diagnosis systems to detect subtle epileptogenic lesions, such as Focal Cortical Dysplasia. Such systems can assist neurologists and neurosurgeons to perform operations with greater precision and fewer side effects.

Medical computer vision applications usually consist of three stages method. The image preprocessing is the first stage, which may enhance features, remove noise and unwanted information by segmenting the Region of Interest (ROI). The second stage is to represent the image into a numerical vector based on features that best describe the content. And finally, a pattern recognition algorithm is used in order to detect patterns and classify new images [Conci et al. 2008].

Thus, our goal with this paper is to develop a classification method in which, based on textural and morphological features extraction, a machine learning algorithm 
can recognize patterns and assist doctors in detecting FCD lesions.

Recent works in the literature approached the FCD classification problem [Antel et al. 2003, Besson et al. 2008, Hong et al. 2014]. In contrast to the actual literature, this work brings a voxel-based classification method that implements a FullyConnected Neural Network, based on Deep Learning approach, to detect patterns based on textural and morphological features. Alongside the Deep Learning approach, multiple traditional classifiers were tested in order to obtain the best method for the proposed extracted features.

Thus, this paper is organized as follows. In Section II, we analyzed the classification methods for FCD detection present on the state of art. Section III described the feature dataset used in this work. Section IV describes the classification method, both based on Deep Learning approach and the traditional machine learning approach. Section $\mathrm{V}$ brings the results obtained and a discussion is made upon observations. Finally, Section VI concludes this work.

\section{FCD Lesions Classification on the Literature}

Detect precisely the epileptogenic region can lead neurosurgeons to stop patient's epileptic seizures. Some authors addressed in the literature the development of automatic classification methods to detect these lesions.

In 2003, [Antel et al. 2003] developed a classification method to detect cortical dysplasia lesions using computational models created from MRI analysis. In their method, it was extracted textural features alongside a Bayesian Classifier. The reported results were around $100 \%$ of specificity and $61 \%$ of sensitivity.

[Besson et al. 2008] developed an automatic detection of FCD for MRI lesions based on morphological features. Their surface-based method with a classifier based on Artificial Neural Network (ANN) achieved an overall accuracy of 87\%.

[Loyek et al. 2008] developed an FCD detection method based on textural features extracted from a voxel perspective. This paper aims to evaluate different texture features to explore which are suitable for detecting epileptogenic lesions. The method was evaluated over five subjects in which the best results were over the fifth patient, with $98 \%$ sensitivity and $92 \%$ specificity.

Later in 2014, [Hong et al. 2014] used textural and morphological features in order to automatically detect cortical dysplasia type-II on MRI. According to the author, using LDA classifier, the proposed method achieved $100 \%$ specificity and $74 \%$ sensitivity.

In 2017, [Adler et al. 2017] proposed a solution for a related problem. On their method, the authors used a novel surface features to detect FCD for pediatric epilepsy. They also compared the obtained results using their novel approach for surface feature extraction with the traditional morphological features. Using an Artificial Neural Network classifier, the authors achieved an AUC of $87 \%$.

After, in 2018, [Jin et al. 2018] proposed a similar work to detect cortical dysplasia lesions using Artificial Neural Network. Their method detected patterns over a morphological dataset with an AUC of $75 \%$. The authors also reported a sensitivity of 


\section{$73.7 \%$ and $90 \%$ specificity.}

[Dev et al. 2019] developed a method to automatically detect FCD regions on MRI using a personalized Fully Convolutional Neural Network based on U-net. Their work achieved a recall of $82.5 \%$, patient-wise.

Recently, in 2020, [Wang et al. 2020] developed a classification method to detect cortical dysplasia. Different from the other methods which use texture and morphological features for feature detection, the authors chose to use Convolutional Neural Networks in order to perform the classification and feature extraction. CNN's have convolutional layers, responsible for extracting features in the form of feature maps. The authors reported an overall accuracy of $85 \%$, a sensitivity of $90 \%$, and a specificity of $99.78 \%$.

\section{Textural and Morphological Features Dataset}

All the features used in this paper were processed, extracted and provided by [Simozo 2018]. The author acquired an MRI with T1, T2, and FLAIR modalities. First, 15 images went through a preprocessing stage. In this stage, the images (i) underwent a correction of the inhomogeneity of the magnetic field, using the N4BiasFieldCorrection (ANTs) method; (ii) went through a process of isolating the cortical surface called skull stripping; (iii) were submitted to a histogram matching process in order to standardize the histogram's intensity levels; and finally (iv) the images went through PeronaMalik filter for noise removal. After this stage, neurologists manually segmented the FCD lesions. According to the authors, the purpose of this manual segmentation was to delimit the regions of the cortex tissue belonging to the FCD lesion in order to correctly extract features. Figure 1 has an example of the preprocessing each image went through before feature extraction.

After this stage, 20 morphological and textural features were extracted by the authors for each voxel of the image and labeled to allow tissue differentiation between health and FCD lesion. The vector descriptor is composed of eight Haralick's textural features, namely: (i) Energy, (ii) Entropy, (iii) Correlation, (iv) Contrast, (v) Cluster Shade, (vii) Cluster Prominence, (viii) Haralick Correlation, alongside the (ix) Intensity Level and the (x) Cortical Thickness, which is a morphological feature, extracted using Freesurfer algorithm. These ten features are then compared to a template of what a healthy cortex would be, so the score-z is calculated for each feature, resulting in a descriptor vector with size 20. The final dataset had 495.306 voxels, 247.653 of each class.

\section{Classification Methodology}

To detect patterns over the features previously described, traditional machine learning algorithms were used in order to evaluate their performance in classifying FCD lesions. Also, a Deep Learning algorithm is designed and compared alongside the traditional methods.

\subsection{Deep Neural Network Design}

The Deep Neural Network developed was based onthe Fully-Connected Neural Network (FCNN) architecture. The FCNN is a powerful architecture to detect complex patterns by mixing up features representing in hierarchical patterns over numerical input vectors. 
Figure 1. The preprocessing steps performed.

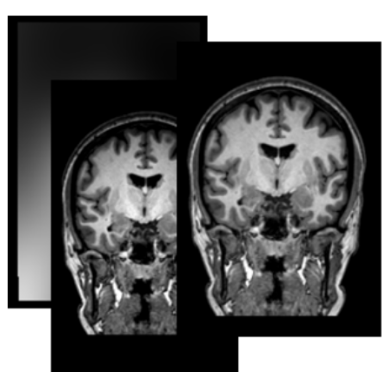

(a)

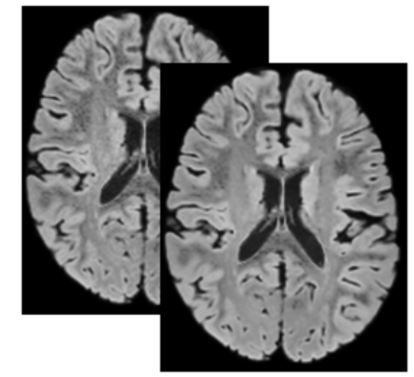

(d)

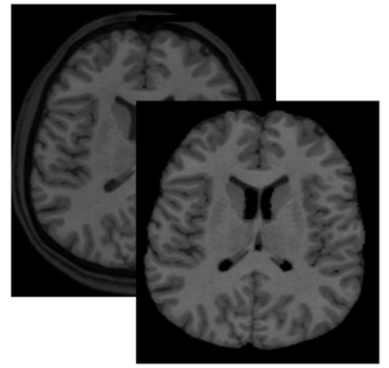

(b)

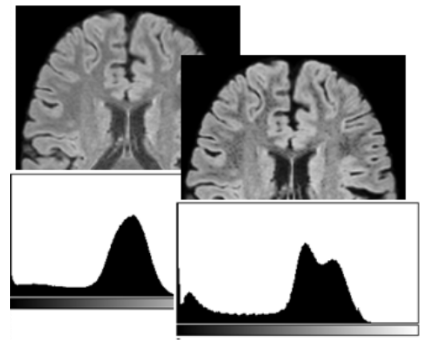

(c)

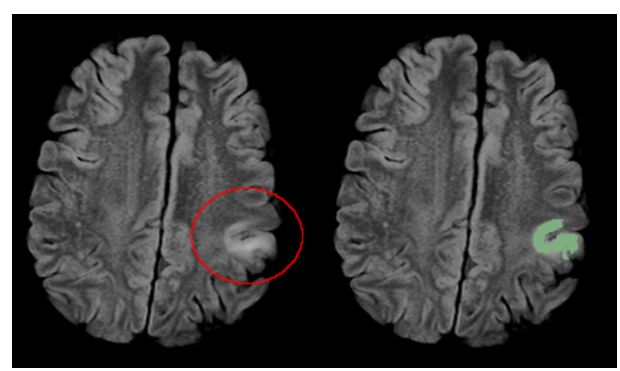

(e)

In (a) inhomogeneity correction using N4 Bias Field Correction, (b) cortical surface isolation through Skull Stripping, (c) intensity histogram normalization using Histogram Match, (d) noise removal through PeronaMalik Filter, and (e) Manual Segmentation. Source: [Simozo 2018].

The proposed Deep Neural Network is modeled as follows. The input layer has 20 neurons to fit all 20 features extracted from MRI. Then, there are three hidden layers, with 16 neurons each, to process and combine hierarchically patterns that best describe and allow an efficient classification. Finally, there is one binary neuron on the output layers, representing the healthy and FCD lesion classes. Figure 2 has a visual representation of the neural network architecture proposed in this work.

In order to set the right hyperparameters, we used the GridSearchCV algorithm distributed on Scikit-Learn framework. This algorithm tests several combinations of hyperparameters such as activation functions, activation function in the output layer, optimizers, and different loss functions.

In this stage, the GridSearchCV searched for three hyperparameters, (i) the activation function used in the hidden layers, (ii) the activation function used in the output layer, and (iii) the optimizer. Four activation functions for the hidden layers were tested, they were the Rectifier Linear Unit (ReLU), Softmax, Sigmoid, and the Exponential Function. In the output activation function, the Sigmoid and Softmax functions were tested. And finally, Adam, RMSprop, and Adadelta optimizers were tested. For each setup, the training algorithm ran for over 100 epochs evaluating the network performance with the validation accuracy. The results of each combination are represented in Table 1.

The final set of hyperparameters, based on the best accuracy obtained during the hyperparameters search, is described as follows. It was used the Rectifier Linear Unity 
Figure 2. The proposed Deep Neural Network architecture.

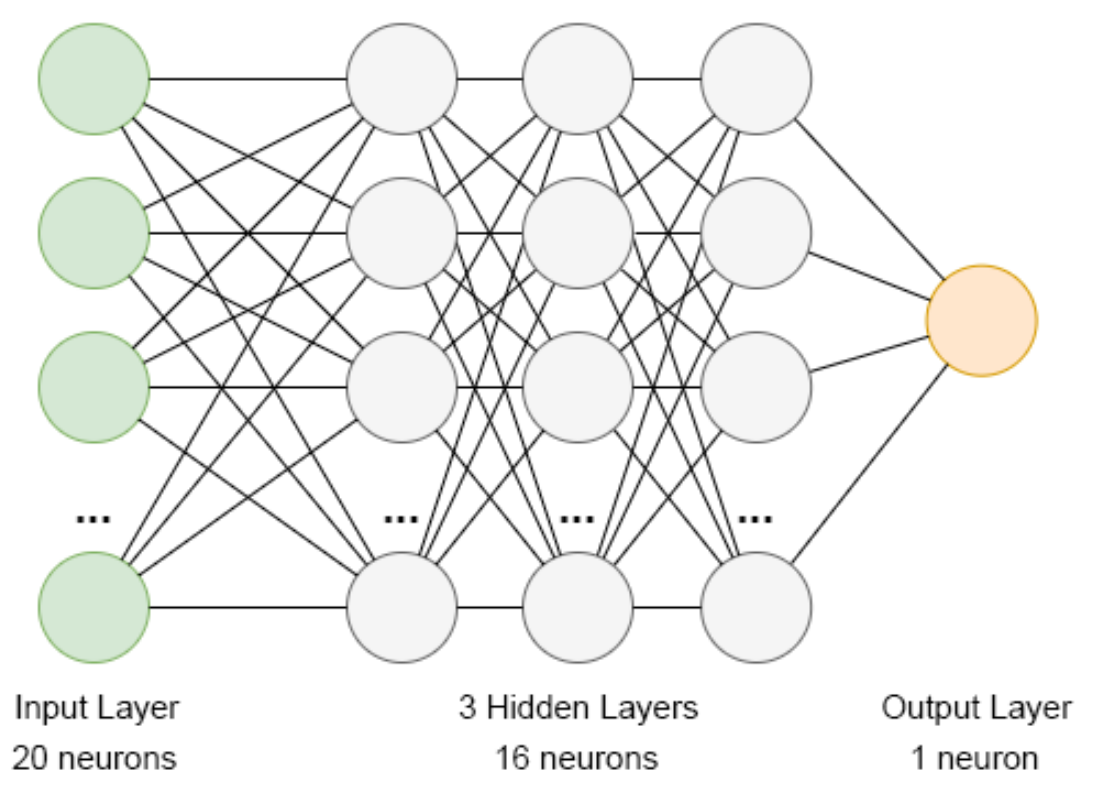

Source: author.

Table 1. Hyperparameters search and tuning results.

\begin{tabular}{llll}
\hline Hidden Layers & Output Layer & Optimizer & Accuracy \\
\hline Exponential & Sigmoid & Adam & $50.13 \%$ \\
Exponential & Sigmoid & RMSprop & $50.13 \%$ \\
ReLU & Sigmoid & RMSprop & $87.57 \%$ \\
Softmax & Sigmoid & Adam & $87.76 \%$ \\
Softmax & Sigmoid & RMSprop & $87.44 \%$ \\
Sigmoid & Sigmoid & Adam & $87.68 \%$ \\
Sigmoid & Sigmoid & RMSprop & $87.25 \%$ \\
ReLU & Sigmoid & Adam & $\mathbf{8 8 . 2 2 \%}$ \\
\hline
\end{tabular}

Source: author.

(ReLU) activation function on the input and hidden layers, while the output layer the Sigmoid activation function was used. To calculate the loss rate it was used the binary crossentropy function. The Adam algorithm was used to optimize the classification method model and to implement its adaptive learning rate. The batch size used had the size of 32 .

Also, in order to avoid overfitting the model into the training set, it was implemented three dropout layers in between the hidden layers. This prevented the model to overfit generalizing the patterns learned by randomly turning off around $20 \%$ neurons on each hidden layer.

\subsection{Other Classifiers}

In order to benchmark the classification performance over the dataset above mentioned, other classifiers were used. These algorithms are based on traditional machine learning, such as Naive Bayes and Logistic Regression, and were developed with Weka 3 Data Mining Software. The following list of algorithms was used during our evaluations: 
1. Bayesian Approach

- Naive Bayes

2. Function Approach

- Logistic Regression

- Multilayer Perceptron (MLP)

3. Lazy Approach

- K-Nearest Neighbours

4. Rules-based Approach

- Decision Table

- One Rule

- Zero Rule

5. Rule-based Approach

- Decision Stump

- Hoeffding Tree

- J48

- Random Tree

- REP Tree

\section{Experiments and Results}

This section is divided into two parts. The first explains how the Deep Neural Network experiments were conducted and its classification results. The second part shows the other classifiers' results to benchmark and rank the classifiers.

In order to compare the results, all experiments followed the k-fold crossvalidation protocol. This protocol separates part of the database under test and training subset so that the data used for training will not be used to evaluate the method. All algorithms used the value 10 as the default value of $k$. Besides, all classifiers were evaluated using similar metrics. The classification accuracy, sensitivity, and specificity were calculated for each approach.

\subsection{Experiments with the deep neural network}

The experiments with FCNN were conducted using Anaconda 3 Data Science Platform, set up with computer vision frameworks, such as OpenCV 4.0, Tensorflow 2.2, ScikitLearn 0.23, and Keras 2.3. All the algorithms were implemented using Python 3.7 programming language. The experiments ran on a computer with Windows 10 Pro operating system, AMD Ryzen 7 processor, 16 GB of RAM and NVIDIA GTX 1060 GPU.

The proposed method was evaluated following the K-Fold Cross-Validation protocol. In this experiment with the FCNN, we set the $k$ value to 10. For each of the ten folds, we calculated five metrics in order to evaluate the method. The metrics calculated were accuracy (ACC), the area under the ROC curve (AUC), sensitivity (SEN), specificity (SPE) and F-Score. By the last experiment, an average between the results obtained was also calculated to represent the classification efficiency. All the results are described in Table 2.

The proposed method obtained an average value of AUC around 97.72\%, a relatively high value of effectiveness to carry out the classification proposed in this work. Also, by analyzing the average sensitivity and specificity, it's verified that both classes are well classified. 
Table 2. Evaluation results obtained from the proposed classification method using K-Fold Cross-Validation.

\begin{tabular}{cccccc}
\hline Fold & ACC & AUC & SEN & SPE & F-Score \\
\hline 0 & $91.60 \%$ & $97.72 \%$ & $90.19 \%$ & $93.02 \%$ & $91.49 \%$ \\
1 & $92.06 \%$ & $97.74 \%$ & $90.25 \%$ & $93.89 \%$ & $91.94 \%$ \\
2 & $92.28 \%$ & $98.89 \%$ & $89.79 \%$ & $94.78 \%$ & $92.10 \%$ \\
3 & $91.93 \%$ & $97.72 \%$ & $89.15 \%$ & $94.67 \%$ & $91.66 \%$ \\
4 & $91.84 \%$ & $97.48 \%$ & $88.86 \%$ & $93.89 \%$ & $91.15 \%$ \\
5 & $91.51 \%$ & $97.62 \%$ & $89.31 \%$ & $94.34 \%$ & $91.60 \%$ \\
6 & $91.95 \%$ & $97.62 \%$ & $88.29 \%$ & $94.75 \%$ & $91.25 \%$ \\
7 & $91.60 \%$ & $97.77 \%$ & $89.11 \%$ & $94.76 \%$ & $91.68 \%$ \\
8 & $91.41 \%$ & $97.36 \%$ & $88.60 \%$ & $94.63 \%$ & $91.39 \%$ \\
9 & $91.17 \%$ & $97.26 \%$ & $88.93 \%$ & $93.87 \%$ & $91.16 \%$ \\
\hline Mean & $\mathbf{9 1 . 7 6 \%}$ & $\mathbf{9 7 . 7 6 \%}$ & $\mathbf{8 9 . 2 5 \%}$ & $\mathbf{9 4 . 2 6 \%}$ & $\mathbf{9 1 . 5 4 \%}$ \\
\hline \multicolumn{5}{c}{ Source: }
\end{tabular}

Source: author.

The algorithm converged around epoch 100-200. Figure 3 illustrates the loss and the validation accuracy curve over the last fold. Note that the accuracy and the loss curve stay stable after epoch 100. Also, the presented curve differs from the overfitting behavior in which the training curve tends to $100 \%$ while the validation curve lowers.

In addition to these experiments, we tested different amounts of neurons in the hidden layers and also the number of inner layers used in the network. It was observed that after the third inner layer, the algorithm hardly converged to accuracy values above $90 \%$, showing no improvements in the classification quality. Lower amounts of 16 neurons and greater than 20 also did not show superior results. This behavior allows us to state that very complex classification models do not allow a more efficient classification and may even result in an inefficient method.

\subsection{Experiments with other classifiers}

The experiments with the other classifiers were conducted using Weka 3 Data Mining Software. The algorithms chosen to be part of this analysis were the algorithms that work well with numerical classification problems, similar to the data represented in the database of textural and morphological features of the voxels. All algorithms followed similar parameters for comparison purposes, such as the number of folds from crossvalidation and the evaluation metrics. The following Table 3 contains the results for each classification method mentioned above.

Analyzing the results obtained, we notice that some approaches have excelled over others. In this way, the J48 and REP Tree algorithms stand out. The J48 is a tree that assembles the nodes based on the best chances of separating the classes correctly given a characteristic. As there is no limit to the number of times a feature can be used, it is expected that this algorithm will be able to create a more efficient path but with risks of overfitting the classification curve since in the experiments, it was observed that the tree built by the algorithm had approximately 3500 leaf nodes. However, to avoid this type of problem, the pruning technique was used in order to generalize the tree, and therefore, the results of this technique are reliable. The REPTree algorithm is based on the same 
Figure 3. The loss and validation accuracy curve over the last fold.

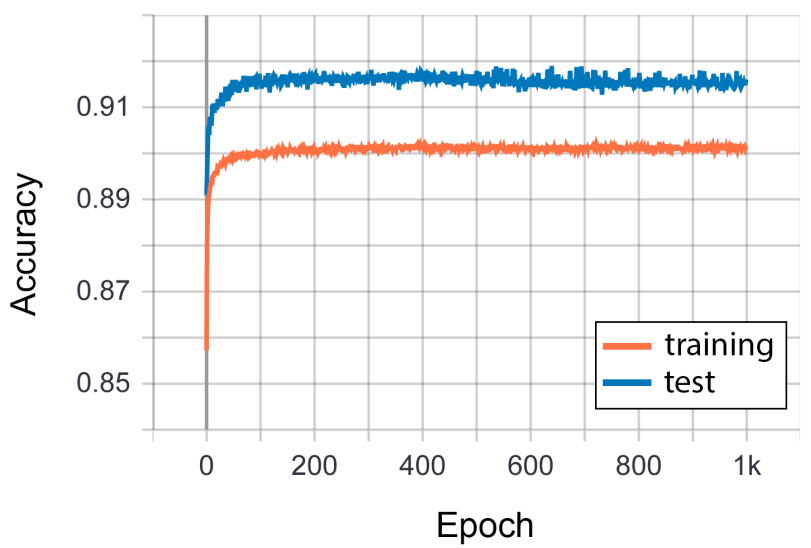

(a)

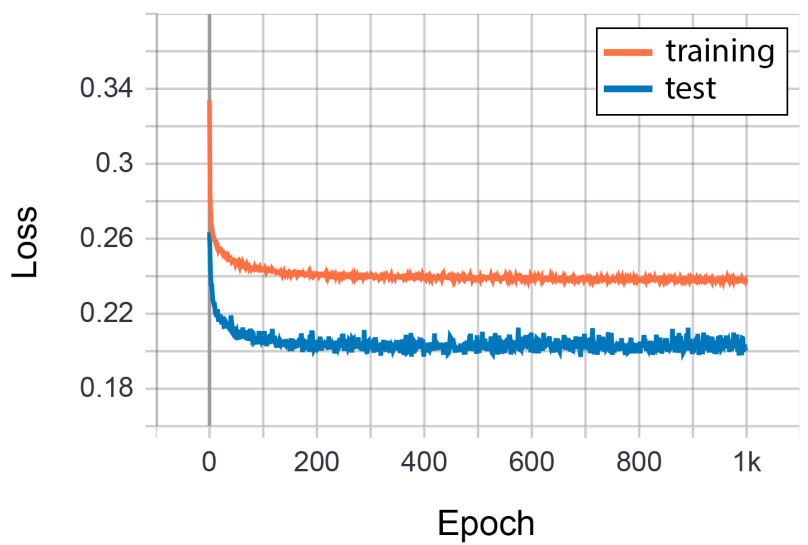

(b)

In (a) the validation accuracy per epoch curve and, in (b) the validation loss per epoch. Source: author.

Table 3. Evaluation results for other classifiers.

\begin{tabular}{cccc}
\hline Algorithm & ACC & SEN & SPE \\
\hline Naive Bayes & $78.51 \%$ & $83.4 \%$ & $73.6 \%$ \\
Logistic Regression & $81.57 \%$ & $85 \%$ & $78.1 \%$ \\
Multilayer Perceptron & $89.86 \%$ & $88.5 \%$ & $91.2 \%$ \\
Decision Table & $87.15 \%$ & $87.7 \%$ & $86.6 \%$ \\
One Rule & $83.62 \%$ & $82.3 \%$ & $84.9 \%$ \\
Zero Rule & $49.99 \%$ & $70 \%$ & $30 \%$ \\
Decision Stump & $78.12 \%$ & $63.2 \%$ & $93 \%$ \\
Hoeffding Tree & $89.76 \%$ & $63.2 \%$ & $93 \%$ \\
J48 & $\mathbf{9 6 . 1 5 \%}$ & $\mathbf{9 6 . 1 \%}$ & $\mathbf{9 6 . 2 \%}$ \\
Random Tree & $94.63 \%$ & $94.7 \%$ & $94.6 \%$ \\
REP Tree & $95.49 \%$ & $95.3 \%$ & $95.7 \%$ \\
FCNN & $\mathbf{9 1 . 7 6 \%}$ & $\mathbf{8 9 . 2 5 \%}$ & $\mathbf{9 4 . 2 6 \%}$ \\
\hline \multicolumn{3}{c}{ Source: author. }
\end{tabular}


previous algorithm, so it is expected that both will have similar results.

\section{Conclusion}

Refractory epilepsy is a specific type of epilepsy that cannot be controlled by normal antiepileptic drugs. This disease is characterized by an excessive neuronal discharge on a certain region of the brain named the epileptogenic zone. Surgical treatments, such as a complete resection or disconnection of this area could lead to the interruption of the crises. In order to assist neurosurgeons to precisely detect the epileptogenic zones, in this paper we proposed a novel approach based on a voxel-level classification using Deep Learning. The proposed method achieved stable and precise results compared to other literature papers.

The thesis that it is possible to identify FCD regions in the cortex by the extracted morphological and textural characteristics is proven due to the detection of patterns performed by several classifiers, even in their different methodologies and approaches. Besides, it is important to note that, although Deep Learning reached results inferior to the tree-based algorithms, it is possible to explore more FCNN architectures and hyperparameters setups. Approaches such as the Very Deep Convolutional Neural Network and three-dimensional Convolutional Neural Networks are also a possibility to deal with MRI volumes. For future work, it is expected to develop a graphic tool for making inferences of exams in order to help neurologists to identify FCD regions in the cortex.

\section{References}

Adler, S., Wagstyl, K., Gunny, R., Ronan, L., Carmichael, D., Cross, J. H., Fletcher, P. C., and Baldeweg, T. (2017). Novel surface features for automated detection of focal cortical dysplasias in paediatric epilepsy. NeuroImage: Clinical, 14:18-27.

Antel, S. B., Collins, D. L., Bernasconi, N., Andermann, F., Shinghal, R., Kearney, R. E., Arnold, D. L., and Bernasconi, A. (2003). Automated detection of focal cortical dysplasia lesions using computational models of their mri characteristics and texture analysis. Neuroimage, 19(4):1748-1759.

Besson, P., Colliot, O., Evans, A., and Bernasconi, A. (2008). Automatic detection of subtle focal cortical dysplasia using surface-based features on mri. In 2008 5th IEEE International Symposium on Biomedical Imaging: From Nano to Macro, pages 16331636. IEEE.

Colombo, N., Tassi, L., Galli, C., Citterio, A., Russo, G. L., Scialfa, G., and Spreafico, R. (2003). Focal cortical dysplasias: Mr imaging, histopathologic, and clinical correlations in surgically treated patients with epilepsy. American Journal of Neuroradiology, 24(4):724-733.

Conci, A., Azevedo, E., and Leta, F. R. (2008). Computação gráfica-teoria e prática,[v. 2]. Rio de Janeiro.

Dev, K. B., Jogi, P. S., Niyas, S., Vinayagamani, S., Kesavadas, C., and Rajan, J. (2019). Automatic detection and localization of focal cortical dysplasia lesions in mri using fully convolutional neural network. Biomedical Signal Processing and Control, $52: 218-225$. 
Fisher, R. S., Acevedo, C., Arzimanoglou, A., Bogacz, A., Cross, J. H., Elger, C. E., Engel Jr, J., Forsgren, L., French, J. A., Glynn, M., et al. (2014). Ilae official report: a practical clinical definition of epilepsy. Epilepsia, 55(4):475-482.

French, J. A. (2007). Refractory epilepsy: clinical overview. Epilepsia, 48:3-7.

Gallucci Neto, J. and Marchetti, R. L. (2005). Aspectos epidemiológicos e relevância dos transtornos mentais associados à epilepsia. Brazilian Journal of Psychiatry, 27(4):323328.

Hong, S.-J., Kim, H., Schrader, D., Bernasconi, N., Bernhardt, B. C., and Bernasconi, A. (2014). Automated detection of cortical dysplasia type ii in mri-negative epilepsy. Neurology, 83(1):48-55.

Jin, B., Krishnan, B., Adler, S., Wagstyl, K., Hu, W., Jones, S., Najm, I., Alexopoulos, A., Zhang, K., Zhang, J., et al. (2018). Automated detection of focal cortical dysplasia type ii with surface-based magnetic resonance imaging postprocessing and machine learning. Epilepsia, 59(5):982-992.

Kwan, P. and Brodie, M. J. (2000). Early identification of refractory epilepsy. New England Journal of Medicine, 342(5):314-319.

Li, L. and Hurford, R. (2020). Journal club: The risk of new-onset epilepsy and refractory epilepsy in older adult stroke survivors. Neurology.

Loyek, C., Woermann, F. G., and Nattkemper, T. W. (2008). Detection of focal cortical dysplasia lesions in mri using textural features. In Bildverarbeitung für die Medizin 2008, pages 432-436. Springer.

Rosenow, F. and Lüders, H. (2001). Presurgical evaluation of epilepsy. Brain, 124(9):1683-1700.

Roy, H., Lippé, S., Lussier, F., Sauerwein, H. C., Lortie, A., Lacroix, J., and Lassonde, M. (2011). Developmental outcome after a single episode of status epilepticus. Epilepsy \& Behavior, 21(4):430-436.

Sheppard, E. and Lippé, S. (2012). Cognitive outcome of status epilepticus in children. Epilepsy research and treatment, 2012.

Simozo, F. H. (2018). Caracterização e identificação de displasias corticais focais em pacientes com epilepsia refratária através de análise de imagens estruturais de ressonância magnética nuclear. $\mathrm{PhD}$ thesis, Universidade de São Paulo.

Taylor, D., Falconer, M., Bruton, C., and Corsellis, J. (1971). Focal dysplasia of the cerebral cortex in epilepsy. Journal of Neurology, Neurosurgery \& Psychiatry, 34(4):369387.

Wang, H., Ahmed, S. N., and Mandal, M. (2020). Automated detection of focal cortical dysplasia using a deep convolutional neural network. Computerized Medical Imaging and Graphics, 79:101662.

WHO, W. H. O. (2019). Oms destaca escassez de tratamento para epilepsia em países de baixa renda. Available at: $<$ https://www.paho.org/bra/index.php?option=com_contentview=articleid=5972 $>$. Accessed in: 15 jul. 2020. 